\title{
Energy-Efficient Resources Management in Container- Based Clouds
}

\author{
Sujalkumar Patel ${ }^{1}$, Dr. Sanjay Patel ${ }^{2}$
}

${ }^{1}$ Department of Computer Engineering, LDRP Institute of Technology and Research, Gandhinagar, Gujarat, India

${ }^{2}$ Assistant Professor, Department of Computer Engineering, LDRP Institute of Technology and Research, Gandhinagar, Gujarat, India

\section{Article Info}

Volume 7, Issue 2

Page Number: 361-365

Publication Issue :

March-April-2021

\section{Article History}

Accepted : 15 April 2021

Published : 20 April 2021

\section{ABSTRACT}

Cloud enables access to a shared pool of virtual resources through Internet and its adoption rate is increasing because of its high availability, scalability and cost effectiveness. However, cloud data centers are one of the fastest-growing energy consumers and half of their energy consumption is wasted mostly because of inefficient allocation of the server's resources. Therefore, this thesis focuses on software level energy management techniques that are applicable to containerized cloud environments. Containerized clouds are studied as containers are increasingly gaining popularity. And containers are going to be major deployment model in cloud environments.

Keywords : Energy Consumption, Cloud, Energy Efficiency

\section{INTRODUCTION}

- Cloud Computing is a realization of utility-oriented delivery of computing services on a pay-as-you-go basis.

$\square$ The National Institute of Standards and Technology (NIST) defines Cloud Computing as “... a model for enabling ubiquitous, convenient, ondemand network access to a shared pool of configurable computing resources (e.g., networks, servers, storage, applications, and services) that can be rapidly provisioned and released with minimal management effort or service provider interaction".

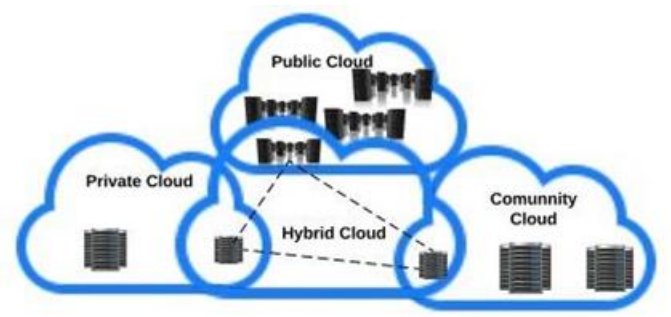

Fig 1.1. The four cloud deployment models: private, public, community, and hybrid cloud
Public: In this model, the Cloud is available to the general public.

Private: The private Cloud is accessible by a business or organization while it is not available to the general public.

Community: This category of cloud provides services to a limited number of individuals or organizations that have shared concerns (e.g., mission, security requirements, and compliance considerations). These organizations are commonly managed, secured, and governed by either a third party managed service provider or all of the participating organizations.

Hybrid: This model is an integration of two or more of the aforementioned cloud deployment models. Here, customers benefit from the multiple deployment models, what consequently eliminates the boundaries and limitations of each cloud model while increasing the capacity through aggregation. 


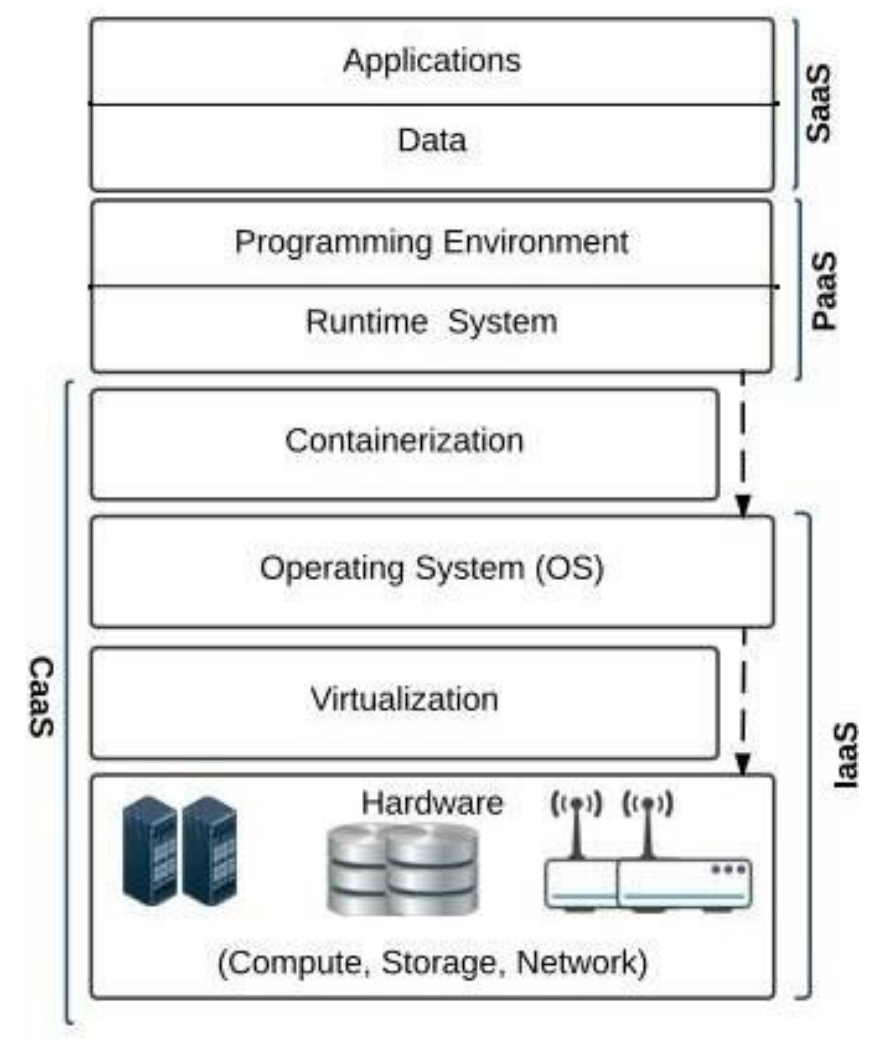

Fig 1.2 Cloud Computing Architecture

\section{Problem Statement}

This thesis tackles research challenges in relation to energy-efficient resource management techniques applicable for containerized cloud environments in which containers are running on VMs. In summary, the following research problems are explored:

How to map tasks/containers to virtual machines considering available cloud workload data?

How workload characterization methodologies/techniques affect the number of identified virtual machine sizes and energy consumption in a $\mathrm{PaaS} / \mathrm{CaaS}$ environment?

How the algorithms applied in various stages of the consolidation process in a CaaS/PaaS environment affects the total energy consumption and SLA violations?

Is container consolidation a better approach than VM consolidation?

\section{PROPOSED METHOD}

Task scheduling algorithms in cloud data center are responsible for allocating the tasks submitted by the different cloud users to the available cloud resources. The main objective of task scheduling algorithm is to achieve a highly performance computing and the best system throughput. Tasks in cloud computing are submitted to the Data Center Broker by the Users. The Data Center Broker works as a mediator between the cloud users and providers and is responsible for scheduling tasks on virtual machines (VM). Data Center is a virtual Infrastructure for housing resources and consists of a number of Hosts. Data center broker provides a policy for scheduling of submitted tasks. Broker communicates directly with the cloud controller and assigns tasks to Virtual machines in the host of the data center. Aws Task Scheduling Processing Way Defined Below.

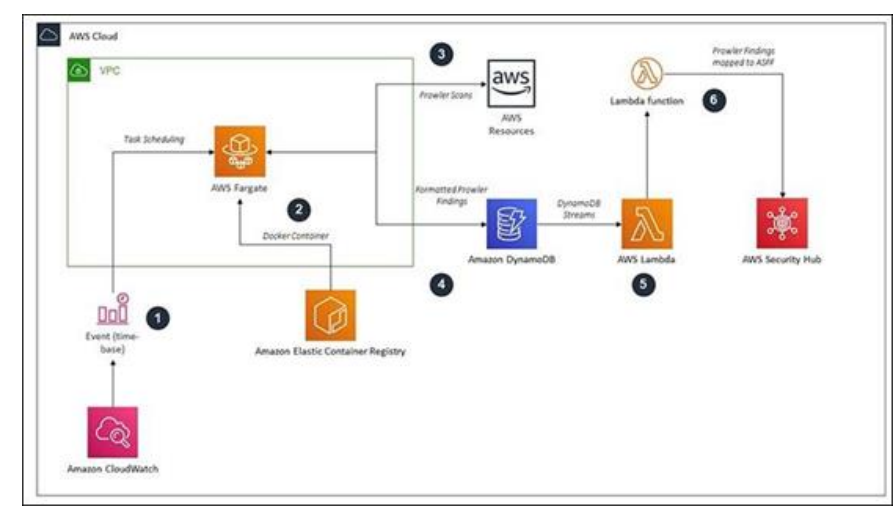

\section{PROPOSED ALGORITHM}

Step -1

Create VM to different Datacenter according to computational power of host/physical server in term of its cost processor, processing speed, memory and storage. 
Step-2

Allocate cloudlet length according to computational power.

\section{Step -3}

Vm Load Balancer maintain an index table of Vms, presently vm has zero allocation.

Step -4

Cloudlet bound according to the length and respective MIPS.

Step -5

Highest length of cloudlet get highest MIPS of virtual machine.

Step -6

Datacenter broker sends the request to the Vm identified with id.

Step -7

Update the available resource.

The Round Robin algorithm focuses on fairness and on distributing the load equally to all nodes. Each job in a queue has same execution time and it will be executed in turn. The scheduler starts assigning VM to each node and move further for next VM to place in next node. Algorithm is applied for all the nodes until one VM is assigned to each node. Again it goes to the first node repeat this process to the next $\mathrm{VM}$ request.

\section{PROPOSED ARCHITECTURE}

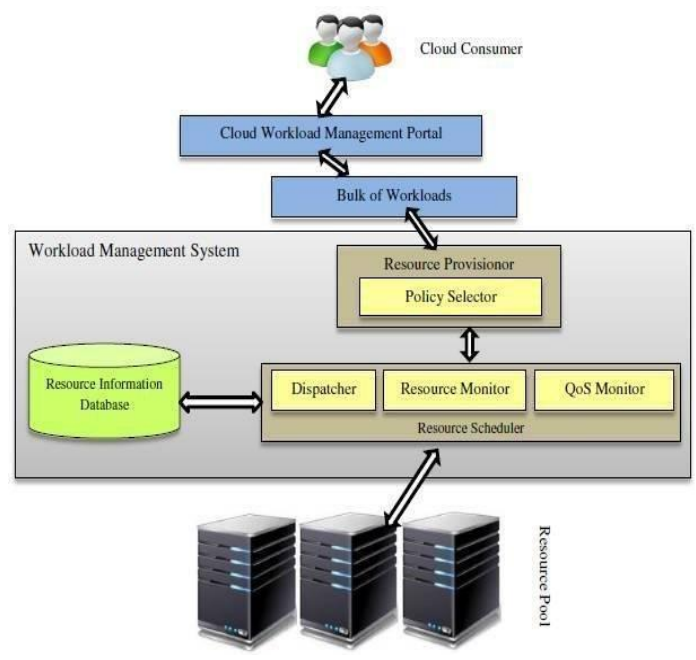

VI. Results

\begin{tabular}{|c|c|c|c|c|c|c|}
\hline \multicolumn{7}{|c|}{ Cloudlet Time Shared Policy } \\
\hline \multirow[t]{2}{*}{ Task } & \multicolumn{3}{|c|}{ Waiting Time } & \multicolumn{3}{|c|}{ Turnaround Time } \\
\hline & FCFS & SJF & $\mathrm{RR}$ & FCFS & SJF & $\mathbf{R} \mathbf{R}$ \\
\hline T1 & 0 & 0 & 0 & 2 & 2.25 & 2 \\
\hline T2 & 0 & 0 & 0 & 4 & 2.36 & 2 \\
\hline T3 & 0 & 0 & 0 & 6 & 2.59 & 2 \\
\hline T4 & 0 & 0 & 0 & 7 & 2.7 & 2 \\
\hline T5 & 0 & 0 & 0 & 8 & 2.81 & 2 \\
\hline T6 & 0 & 0 & 0 & 9 & 2.81 & 2 \\
\hline T7 & 0 & 0 & 0 & 10 & 3.04 & 2 \\
\hline T8 & 0 & 0 & 0 & 11 & 3.15 & 2 \\
\hline T9 & 0 & 0 & 0 & 13 & 3.15 & 2 \\
\hline T10 & 0 & 0 & 0 & 15 & 3.6 & 2 \\
\hline AVG & 0 & 0 & 0 & 8.5 & 2.85 & 2 \\
\hline
\end{tabular}

Task Scheduling TimeShared

Comparison chart for the method

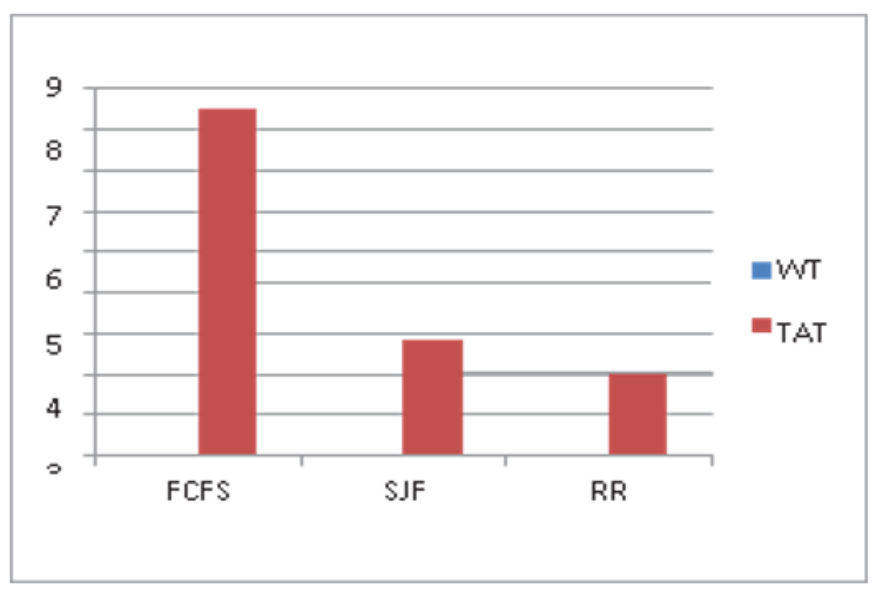

Comparison of FCFS, SJF and RR using cloudlet time shared policy

\section{CONCLUSION}

At the end of the literature survey it can be concluded that Improving the energy efficiency of cloud data centers is an ongoing challenge that can increase the cloud providers return of investment (ROI) and also decrease the $\mathrm{CO} 2$ emissions that are accelerating the global warming phenomenon. Despite the increasing 
popularity of Container as a Service (CaaS), energy efficiency of resource management algorithms in this service model has not been deeply investigated.

\section{REFERENCES}

[1]. Cloud computing" webpage on Wikipedia OnlineAvailable: http://en.wikipedia.org/wiki/Cloud_computing

[2]. M. Armbrust, A. Fox, R. Griffith, A. D. Joseph, R. Katz, A. Konwinski, G. Lee, D. Patterson, A. Rabkin, I. Stoica et al., "A view of cloud computing," Communications of the ACM, vol. 53, no. 4, pp. 50-58, 2010.

[3]. B. Arnold, S. A. Baset, P. Dettori, M. Kalantar, I. I. Mohomed, S. J. Nadgowda, M. Sabath, S. R. Seelam, M. Steinder, M. Spreitzer, and A. S. Youssef, "Building the ibm containers cloud service," IBM Journal of Research and Development, vol. 60, no. 2-3, pp. 9:1-9:12, March 2016.

[4]. A. Beloglazov and R. Buyya, "Energy efficient allocation of virtual machines in cloud data centers," in Proceedings of the 10th IEEE/ACM International Conference on Cluster, Cloud and Grid Computing (CCGrid 2010), May 2010, pp. 577-578.

[5]. A. Beloglazov, R. Buyya, Y. C. Lee, A. Zomaya et al., "A taxonomy and survey of energyefficient data centers and cloud computing systems," Advances in computers, vol. 82, no. 2, pp. 47-111, 2011.

[6]. M. Blackburn, Five ways to reduce data center server power consumption. The Green Grid, 2008.

[7]. R. Buyya, A. Beloglazov, and J. Abawajy, "Energy-efficient management of data center resources for cloud computing: a vision, architectural elements, and open challenges," in Proceedings of 16th International Conference on Parallel and Distributed Processing
Techniques and Applications (PDPTA 2010), July 2010, pp. 6- 17.

[8]. Y. Chen, S. Alspaugh, D. Borthakur, and R. Katz, "Energy efficiency for large- scale MapReduce workloads with significant interactive analysis," in Proceedings of the 7th ACM European Conference on Computer Systems, April 2012, pp. 43-56.

[9]. Sareh Fotuhi Piraghaj, Energy-Efficient Management of Resources in Enterprise and Container-based Clouds, Ph.D. Thesis, The University of Melbourne, Australia, March 2016.

[10]. A. Verma, G. Dasgupta, T. K. Nayak, P. De, and R. Kothari, "Server workload analysis for power minimization using consolidation," in Proceedings of the 2009 Conference on USENIX Annual Technical Conference, 2009, pp. 28-28.

[11]. https://console.aws.amazon.com/

[12]. D. Shepherd, "Containers as a Service (CaaS) is the cloud operating system - i build the cloud," http://www.ibuildthecloud.com/blog/2014/08/1 9/containersas-a- service-caas-is-the-cloudoperating-system/, (Accessed on 03/01/2016).

[13]. G. Nagy, "Operating system containers vs. application containers," https://blog.risingstack.com/operating-systemcontainers-vs-application-containers/, May 2015, (Accessed on 10/22/2015).

[14]. R. Morabito, "Power consumption of virtualization technologies: an empirical investigation," in Proceedings of the 2015 IEEE/ACM 8th International Conference on Utility and Cloud Computing (UCC), December 2015, pp. 522-527.

[15]. V. Mohan Raj and R. Shriram, "Power aware provisioning in cloud computing environment," in Proceedings of the 2011 International Conference on Computer, Communication and Electrical Technology (ICCCET), March 2011, pp. 6-11. 
[16]. Y. C. Lee, H. Han, A. Y. Zomaya, and M. Yousif, "Resource-efficient workflow scheduling in clouds," Knowledge-based Systems, vol. 80, pp. 153-162, 2015.

[17]. K. H. Kim, A. Beloglazov, and R. Buyya, "Power-aware provisioning of cloud resources for real-time services," in Proceedings of the 7th International Workshop on Middleware for Grids, Clouds and e-Science (MGC 2009). Champaign, USA: ACM, New York, USA, November 2009, pp. 1:1-1:6.

[18]. J. Jiang, T. Lan, S. Ha, M. Chen, and M. Chiang, "Joint VM placement and routing for data center traffic engineering," in Proceedings of the 2012 IEEE International Conference on Computer Communications (INFOCOM 2012), March 2012, pp. 2876-2880.

\section{Cite this article as :}

Sujalkumar Patel, Dr. Sanjay Patel, "Energy-Efficient Resources Management in Container- Based Clouds", International Journal of Scientific Research in Computer Science, Engineering and Information Technology (IJSRCSEIT), ISSN : 2456-3307, Volume 7 Issue 2, pp. 361-365, March-April 2021. Available at doi : https://doi.org/10.32628/CSEIT217278

Journal URL : https://ijsrcseit.com/CSEIT217278 